\title{
Dynamic Molecular Imaging for Hepatic Function Assessment in Mice: Evaluation in Endotoxin-Induced and Warm Ischemia-Reperfusion Models of Acute Liver Failure
}

Félicie Sherer ${ }^{1 *}$, Gaetan Van Simaeys ${ }^{1}$, Jesper Kers ${ }^{2,3}$, Qing Yuan ${ }^{2,4}$, Gilles Doumont ${ }^{1}$, Marie-Aline Laute ${ }^{1}$, Cindy Peleman ${ }^{5}$, Dominique Egrise ${ }^{1}$, Tony Lahoutte $^{5}$, Véronique Flamand ${ }^{2 \#}$ and Serge Goldman ${ }^{1 \#}$

${ }^{1}$ Center for Microscopy and Molecular Imaging (CMMI) and Department of Nuclear Medicine, Erasme Hospital, Université Libre de Bruxelles (ULB), Belgium

${ }^{2}$ Institute for Medical Immunology, Université Libre de Bruxelles (ULB), Gosselies, Belgium

${ }^{3}$ Department of Pathology, Academic Medical Center, Amsterdam, The Netherlands

${ }^{4}$ Organ Transplant Center, The 309th Hospital of PLA, Beijing, China

${ }^{5}$ In vivo Cellular and Molecular Imaging (ICMI) Laboratory, Vrije Universiteit Brussel (VUB), Brussels, Belgium

\#The authors contributed equally to the work

*Corresponding author: Félicie Sherer, CMMI, 8 rue Adrienne Bolland, B-6041 Gosselies, Belgium, Tel: +32 26509789 ; Fax:+32 2 650 9795; E-mail: fsherer@ulb.ac.be

Received September 29 2014, Accepted November 14 2014, Published November 192014

Copyright: $\odot 2015$ Sherer F, et al. This is an open-access article distributed under the terms of the Creative Commons Attribution License, which permits unrestricted use, distribution, and reproduction in any medium, provided the original author and source are credited.

\begin{abstract}
Background: In hepatic transplantation, inflammatory response related to liver ischemia-reperfusion injury is an important cause of hepatocellular damage that may lead to organ dysfunction. This project aims to develop a new method of dynamic imaging for the local analysis of hepatic function using un-metabolized 99mTc-labeled mebrofenin excretion time in the bile canaliculi as a read-out.

Methods: C57BL/6 female mice underwent acute liver damage induced either by endotoxin administration or by warm ischemia-reperfusion. Liver damage intensity was assessed with a 99mTc-labeled mebrofenin dynamic planar imaging protocol, together with biological parameters of liver damage - levels of blood transaminases, liver necrosis and neutrophil infiltration. The acquisition data consisted of a series of 60 -frame pinhole images performed on a gamma camera. A region of interest was drawn within the hepatic area in order to measure liver activity on each frame. Excretion rate was quantified as the time necessary for the count value to reach $50 \%$ (T0.5Exc) and $20 \%$ (T0.2Exc) of the maximum liver count value. We compared biological parameters of liver damage - levels of blood transaminases, liver necrosis and neutrophil infiltration — with $99 \mathrm{mTc}$-labeled mebrofenin excretion times in both models of liver damage and in control animals.
\end{abstract}

Results: 99mTc-labeled mebrofenin excretion times (T0.5Exc and T0.2Exc) were significantly increased in both models of liver damage.

Conclusions: We concluded that quantification of liver function is feasible in mice using dynamic planar pinhole imaging with $99 \mathrm{mTc}$-mebrofenin as tracer of the hepato-biliary function. This method is particularly suited to the noninvasive evaluation of immune and pharmacological interventions aiming at a reduction of early liver insults related to ischemic-reperfusion phenomenon.

Keywords: Molecular imaging; Liver; Ischemia-reperfusion; Mebrofenin
Abbreviations
DAMPs: Damage Associated Molecular Pattern molecules; ET: EndoThelin; HIDA: Hepatic IminoDiacetic Acid; HMGB-1: the nuclear protein High Mobility Group Box 1; IRI: Ischemia- Reperfusion Injury; IR2h: group of mice imaged 2 hours after Ischemia-Reperfusion; IR24h: group of mice imaged 24 hours after Ischemia-Reperfusion; LPS: Lipopolysaccharide; MRP: Multidrug Resistance Proteins; NO: Nitric Oxide; NLRs: NOD-Like Receptor pathways; OATP: The Main Salt and Organic Anion Transporters; PAMPs: Pathogen Associated Molecular Pattern molecules; RNS: Reactive Nitrogen Species; ROS: Reactive Oxygen Species; sALT:

serum Alanine AminoTransferase; sAST: serum Aspartate AminoTransferase; SPECT: Single Photon Emission Computed Tomography; TLR: Toll-Like Receptor; TLRs: Toll-Like Receptor pathways

\section{Introduction}

Ischemia-reperfusion injury (IRI) refers to the cellular damage induced by oxygen delivery restoration in an organ subjected to a hypoxic insult [1]. Liver IRI occurs in many common clinical situations including extensive liver injury, major liver resection, haemorrhagic shock and liver transplantation. Postsurgical liver dysfunction or failure is strongly linked to the extent of hepatic IRI and is an expected consequence of these heavy liver surgical procedures [2]. Bacterial cell wall lipopolysaccharide (LPS) is a typical 
agent used in animal models of endotoxin-induced liver injury [3] Indeed, LPS is a trigger for cytokine formation through the activation of the TLR-4 pathway; at a high dose it directly activates the complement system. The cytokines secreted by the LPS-activated Kupffer cells, and the activated complement fragments, prime neutrophils to enhance ROS production [4]. Ultimately, the relevant effects of inflammation-induced liver insults result in an impairment of hepatic functions. New methods of liver function evaluation, particularly in vivo non-invasive methods such as molecular imaging, are therefore needed to get insight into the early local metabolic consequences of liver injury. The $99 \mathrm{mTc}$-radiolabeled lidocaine analogue mebrofenin (99mTc-labeled mebrofenin) is excreted unmetabolized in the bile canaliculi [5-9]. Taking profit of its rapid hepatic extraction, this hepatic iminodiacetic acid (HIDA) tracer is routinely used for the evaluation of bile excretion in clinical practice $[10,11]$. This tracer is specific for hepatocellular transport and it has low competition effect for hepato-biliary excretion of bilirubin [12]. It has already been used in animal models, in particular the blood clearance of this compound has been tested in a canine model of toxic liver injury and in a rabbit model of IRI in which excretion of the tracer has been assessed [13,14]. Obviously, the opportunities offered by mice models are wider than those of the larger animal models, in particular for what concerns immunological interventions and very recently, the precise mechanisms of hepatic uptake and biliary excretion of $99 \mathrm{mTc}$-labeled mebrofenin have been studied in mice $[15,16]$. The hepatic uptake of this tracer occurs via a sodiumindependent transport mechanism involving the organic anion transport protein $1 \mathrm{a}$ and $1 \mathrm{~b}(\mathrm{Oatp} 1 \mathrm{a} / \mathrm{lb})$, and excretion into the bile occurs through multidrug resistance proteins (mrp) 2 and 3 [16].

In this study, we present a quantitative assessment of the hepatic excretory function using dynamic imaging of $99 \mathrm{mTc}$-labeled mebrofenin hepatic excretion in two different murine models of liver damage, one based on a single injection of LPS, the other based on a warm liver IRI.

\section{Materials and Methods}

\section{Mice}

8-12 week-old female wild-type C57BL/6 mice $(\mathrm{n}=65)$ were purchased from Harlan (Horst, The Netherlands). Mice were housed and bred in our specific pathogen-free animal facility. All animal studies were approved by the Institutional Animal Care and local committee for animal welfare.

\section{Endotoxin-induced liver dysfunction}

We studied the well-known model of endotoxin-induced sepsis, which results in a profound multi-organ response that includes the liver [3,17]. Mice received a single $200 \mu$ intravenous injection of purified LPS ( $1 \mathrm{~g} / \mathrm{L}$ LPS in $0.9 \% \mathrm{NaCl}$; LPS, Ultra-Pur, CaylaInVivoGen, Toulouse, France). As a control, mice received a $200 \mu \mathrm{l}$ intravenous injection of $0.9 \% \mathrm{NaCl}$. All mice were supplemented with an additional subcutaneous $100 \mu \mathrm{l} 0.9 \% \mathrm{NaCl}$ injection for extra hydration. The hepato-biliary functional imaging was performed 48 hours after LPS administration.

\section{Liver ischemia-reperfusion injury}

Mice were anesthetized with $4 \%$ isoflurane in $1 \mathrm{~L} / \mathrm{min}$ oxygen flow induction and $2-3 \%$ isoflurane in $1 \mathrm{~L} / \mathrm{min}$ oxygen flow as maintenance inhalation (Matrx VIP 3000 veterinary vaporizer, Midmark Corporation, Versailles, USA and an oxygen concentrator, Oxymat 3, Weinmann, Hamburg Germany). During the entire experiment until wake-up, the body temperature of the mice was kept at $36-37^{\circ} \mathrm{C}$ by a homeostatic control unit (Harvard Apparatus, Holliston, United States of America). Under a microsurgical microscope (Wild Heerbrugg, Gais, Switzerland), a mid-line laparotomy, up till the sternum was performed in order to visualize the portal triad. With saline-soaked cotton-wool sticks, the portal triad in between the cranially-located right/left lobe and the caudally-located caudate and the quadrate lobe was localized and liberated from connecting liver ligaments. A nontraumatic vascular Bulldog clamp was placed on the portal triad in order to occlude the hepatic oxygen and nutrient supply for 60 minutes. In this way, the intestinal blood in the portal vein can still be exported towards the caudate and quadrate lobes and lethal venous congestion in the intestine does not occur ( 30\% non-ischemic hepatic area is remaining) $[1,18]$. After 60 minutes of hepatic occlusion, paling of the liver was validated and reperfusion was initiated by removing the vascular clamp. Regaining of intra-hepatic circulation was visually verified in each mouse. Additionally, 200 $\mu \mathrm{l}-0.9 \% \mathrm{NaCl}$ was subcutaneously injected for hydration. The hepatobiliary functional imaging was performed at 2 hours and 24 hours after reperfusion. Sham-operated mice underwent the same procedure except for the clamp placement. Serum aminotransferase (sALT and sAST) levels were measured using an auto analyzer (Modular P800, Hitachi).

\section{Histology}

Formalin-fixed tissues samples were embedded in paraffin. Liver sections $(4 \mu \mathrm{m})$ were stained with hematoxylin/eosin. Three histological lesions in liver ischemia-reperfusion injury were scored according to the methods described by Suzuki and colleagues [19], which included venous congestion, vacuolization and necrosis all ranked in a scale from 0 to 4 . In the LPS model, immunohistology was performed for neutrophil staining using rat anti-Ly-6G monoclonal antibodies (BD Biosciences) and Biotin-conjugated Goat anti-rat IgG (Jackson Immunoresearch laboratories) as secondary antibodies.

\section{Hepato-biliary functional imaging}

We developed an imaging protocol for bile flow dynamic analysis using 99mTc-labeled mebrofenin [6,9]. The 99mTc-labeled mebrofenin tracer was produced using the Bridatec human kit of GE Healthcare (Diegem, Belgium) and $99 \mathrm{mTc}$ eluted from a radioisotope generator (Covidien, Petten, The Netherlands), according to the standard procedure proposed by the provider. The obtained $99 \mathrm{mTc}-$ labeled mebrofenin was diluted in the same volume of $0.9 \% \mathrm{NaCl}$. A mean \pm standard deviation of $0.94 \pm 0.25 \mathrm{mCi} 99 \mathrm{mTc}$-mebrofenin was finally injected through the tail-vein of the mice. Ten minutes prior to acquisition, the mice included in the endotoxin protocol were anesthetized by a single intra-peritoneal injection of a combination of xylazine (Rompun $23,32 \mathrm{mg} / \mathrm{mL}$ ) and ketamine (Ketalar $50 \mathrm{mg} / \mathrm{mL}$ ) diluted in $0.9 \% \mathrm{NaCl}$ in a ratio of 1:2:7 (no isoflurane anesthesia station available at this time). The mice included in the liver IRI protocol underwent another round of isoflurane anaesthesia as described in the aforementioned section at 2 hours (IR2h) or 24 hours (IR24h) post-reperfusion for imaging purpose. The 99mTc-labeled mebrofenin was injected under the single photon emission computed tomography (SPECT) camera in order to start the imaging acquisition at the time of injection. Liver function was evaluated by the capacity of 
Citation: Sherer F, Van Simaeys G, Kers J, Yuan Q, Doumont G, et al. (2015) Dynamic Molecular Imaging for Hepatic Function Assessment in Mice: Evaluation in Endotoxin-Induced and Warm Ischemia-Reperfusion Models of Acute Liver Failure. J Liver 4: 170. doi: $10.4172 / 2167-0889.1000170$

Page 3 of 7

the liver cells to secrete the tracer in the biliary system. We observed a global death rate of about $23 \%$.

\section{Image acquisition}

Dynamic planar pinhole imaging of mice included in the endotoxin protocol was performed using a modified clinical gamma camera (e.cam180 Siemens Medical Solutions, Wheaton, IL, USA) equipped with a single pinhole collimator (pinhole opening $1.5 \mathrm{~mm}$, focal length $250 \mathrm{~mm}$ ). Dynamic planar pinhole imaging of mice included in the liver IRI protocol was performed using a camera (nanoSPECT In Vivo Preclinical Imager, Bioscan, Washington DC, USA) equipped with single pinhole collimator (pinhole opening $1.5 \mathrm{~mm}$, focal length $45 \mathrm{~mm}$ ). When isoflurane was used for anesthesia, the mice were laid down in a temperature-controlled $\left(37^{\circ} \mathrm{C}\right)$ imaging bed (Minerve, France) with the head put in the anesthesia mask. A 20-minute acquisition of 60 planar frames ( 40 of 15 s, 20 of 30 s; 256 by 256 pixel image matrix; zoom $=2$ for LPS and $z o o m=1.4$ for IRI) was then performed for each mouse, in the ventral position, starting at tracer injection.

\section{Data analysis}

A 2D region of interest (ROI) was drawn with OsiriX (http:// www.osirix-viewer.com) within the hepatic parenchyma and the total counts inside the ROI were measured on each time frame. In each mouse, two elliptical ROIs were drawn, one on the left side and one on the right side of the liver parenchyma. Excretion curves are timeactivity curves (TAC) obtained from both ROIs were linearly interpolated to get one point every second from $0 \mathrm{~s}$ to $1185 \mathrm{~s}$, and then smoothed using a 5-point moving average (Matlab, The MathWorks, Inc., USA). As our methodology did not give us access to an imagebased blood input function, we did not apply any deconvolution for the vascular phase on the TAC. However, the impact of this deconvolution is major on the (early) uptake phase of the tracer, but very limited on the excretion phase. In order to quantitatively estimate the excretion function and its possible alteration, we then determined the value and the time at which peak $99 \mathrm{mTc}$-mebrofenin uptake is observed, as well as the times necessary for the count rate value to reach $50 \%$ (T0.5Exc) and $20 \%$ (T0.2Exc) of the observed maximum liver count rate value. Since no left/right differences were found significant, averaged values are reported and used for statistical inference purpose. In mice for which $50 \%$ or $20 \%$ of the maximum liver count value was not reached at the end of the 20-minute scan, corresponding values were set conservatively at the total scan time 1200 s and twice this value 2400s, respectively. These conservative values are thus underestimated compared to their expected values. Yet, these expected values were computed when possible, i.e. when the $99 \mathrm{mTc}$-mebrofenin excretion phase was significantly initiated to allow the computation, by means of a numerical extrapolation based on a nonlinear regression of the logarithm count rate data using Matlab curve fitting tools (Matlab 7.6, The MathWorks, Inc., Natick, MA, USA).

\section{Statistical analysis}

Data of liver damage parameters are expressed as mean \pm SEM for the biological and histological analysis, whereas for the imaging data analysis excretion times are expressed as median $\pm 95 \%$ confidence interval. Statistical analysis of data was realized by using a one-tailed non parametric Mann-Whitney test. Reproducibility of the excretion curves was assessed for each group (LPS, control, liver IRI and sham) on the basis of the coefficient of variation CV: Inter-curve coefficients of variation (CVs) for $50 \%$ and $20 \%$ excretion times were analyzed. The $\mathrm{H} 1$ hypothesis that both endotoxin-induced liver dysfunction and hepatic ischemia-reperfusion injury result in impaired excretion of 99mTc-labeled mebrofenin, hence in longer excretion times, was validated by one-tailed non-parametric Mann-Whitney-Wilcoxon tests. For this purpose, we used the conservative values of excretion times, i.e. the total time of the acquisition, which is known to be underestimated for some of the sick mice. A Receiver Operating Characteristics (ROC) analysis tests the ability of various threshold values to discriminate between two conditions. It also allows defining the threshold value with the highest specificity and sensitivity, i.e. the cut-off value. On the basis of this statistical approach, we further assessed the ability of $99 \mathrm{mTc}$-labeled mebrofenin dynamic planar imaging of excretion, i.e. T0.5Exc and T0.2Exc, to discriminate between endotoxin-induced and IRI-induced liver dysfunction on the one side and their respective controls on the other side. For the ROC analyses, we only included mice for which the excretion times were observed or could be computed by means of numerical extrapolation. The conservative values would indeed lead to biased results. The pvalues below 0.05 were considered statistically significant throughout the study.

\section{Results}

\section{Intravenous injection of LPS prolongs hepatic excretion of 99mTc-labeled mebrofenin}

We first evaluated liver injury caused by LPS administration as commonly performed. Intravenous injection of $200 \mu \mathrm{L}$ of a LPS solution of $1.0 \mathrm{~g} / \mathrm{L}$ (i.e. approximately $10 \mathrm{mg} / \mathrm{kg}$ with mice of $20 \mathrm{~g}$ body weight) significantly increased serum ALT and AST levels 6 hours post treatment (Figure 1A). Liver neutrophil infiltration was observed in a LPS dose-dependent manner 24 hours post injection compared with control livers (Figure 1B).

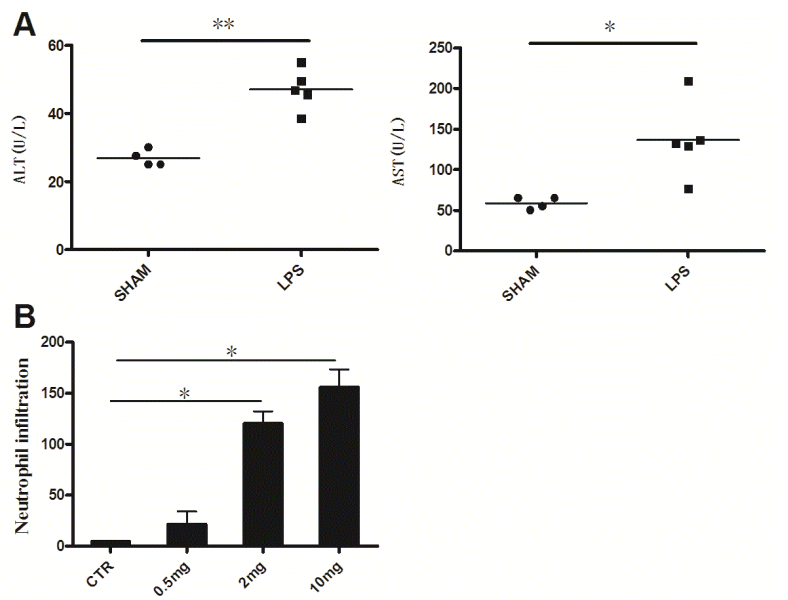

Figure 1: Liver pathology induced by LPS. (A) Serum ALT and AST levels from C57BL $/ 6$ mice, 6 hours after $10 \mathrm{mg} / \mathrm{kg}$ LPS administration $(n=5)$ and in SHAM vehicle controls $(n=4)$. (B) Neutrophil infiltration count $/ \mu \mathrm{m}^{2}$ liver section determined by antiLy-6G immunostaining 24 hours post $0.5,2$ or $10 \mathrm{mg} / \mathrm{kg}$ LPS treatment. Results are mean values $\pm \operatorname{SEM}\left({ }^{\star} \mathrm{p}<0.05,{ }^{\star *} \mathrm{p}<0.01\right)$. 
Citation: Sherer F, Van Simaeys G, Kers J, Yuan Q, Doumont G, et al. (2015) Dynamic Molecular Imaging for Hepatic Function Assessment in Mice: Evaluation in Endotoxin-Induced and Warm Ischemia-Reperfusion Models of Acute Liver Failure. J Liver 4: 170. doi: $10.4172 / 2167-0889.1000170$

Page 4 of 7

We then performed the liver imaging 48 hours after LPS treatment Both characteristic excretion times (T0.5Exc and T0.2Exc) were increased in mice treated with LPS compared with the control animals (Figure 2A and 2B). Four LPS animals never reached $20 \%$ of the maximum liver count value at the end of the scan, among which one animal never reached $50 \%$ of this count value. The T0.5Exc were $224 \mathrm{~s}$ (171-460 s) [median (95\% CI)], $180 \mathrm{~s}$ (131-208 s) in LPS and control group, respectively $(\mathrm{p}=0.01)$. The T0.2Exc were $534 \mathrm{~s}(440-1188 \mathrm{~s})$ and $401 \mathrm{~s}(332-428 \mathrm{~s})$ in LPS and control group, respectively $(\mathrm{p}=0.002)$ (Figure $2 \mathrm{~A}$ and $2 \mathrm{~B})$.
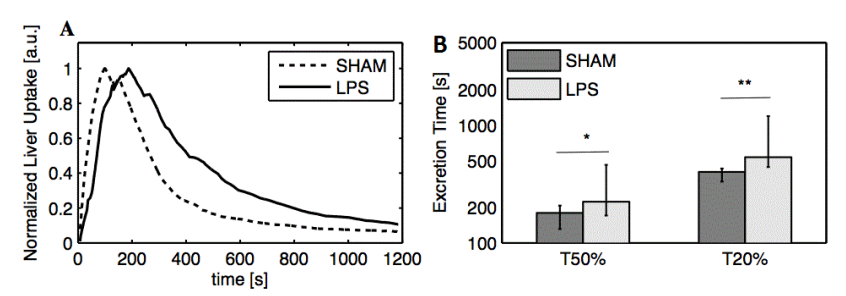

Figure 2A and 2B: Liver excretion of 99mTc-labeled mebrofenin after LPS treatment. (A) Excretion curves for LPS treated mice (solid line) and SHAM treated mice (dashed line), obtained by taking the median value at each time point from the dynamic planar acquisitions, the arbitrary units were normalized to 1 relative to the corresponding peak value in each group. (B) T0.5Exc and T0.2Exc values from $10 \mathrm{mg} / \mathrm{kg}$ LPS treated mice $(\mathrm{n}=11)$ and SHAM treated mice $(n=9)$ at 48 hours. Results are median values $\pm 95 \%$ CI. $\left({ }^{*} \mathrm{p}<0.05,{ }^{* *} \mathrm{p}<0.01\right)$
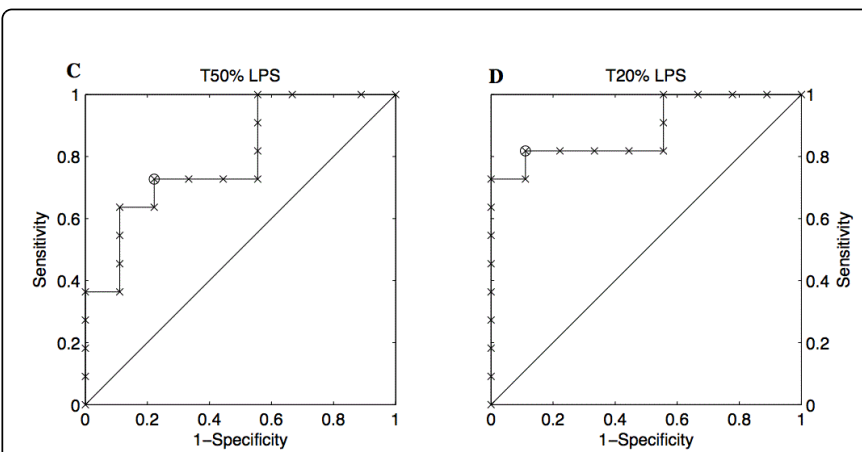

Figure 2C and 2D: (C) ROC curves for T0.5Exc for LPS vs. control. (D) ROC curves for T0.2Exc for LPS vs. control. The more the curve approaches the point $(x=0 ; y=1)$, the higher is the discriminating power of the test, T0.2Exc is the best to discriminate between LPS-treated mice and SHAM treated mice.

Individual excretion times are shown in Table 1. The CV for the T0.5Exc of LPS and control treated mice was $43 \%$ and $20 \%$ respectively while the CV for the T0.2Exc of LPS and control treated mice was $17 \%$ and $13 \%$ respectively, indicating that the T0.2Exc variable was the most reproducible among the mice in the LPS model. The ROC curve performed on T0.5Exc showed an area under the curve (AUC) of 0.80 (SE: $0.10,95 \%$ CI: $0.60-1.00$ ), which proved to be statistically greater than 0.5 (1-tailed $\mathrm{p}<0.001)$ in LPS versus control animals (Figure 2C). The cut-off value for T0.5Exc was $198 \mathrm{~s}$. The accompanying sensitivity was $73 \%$ (95\% CI: 46\%-99\%) and the specificity was $78 \%$ (95\% CI: 46\%-99\%). A similar analysis performed on T0.2Exc (Figure 2D) led to an AUC of 0.89 (SE: 0.08, 95\% CI: $0.74-1.00$ ), which proved to be statistically greater than 0.5 (1-tailed $\mathrm{p}<0.001)$. The cut-off value for T0.2Exc was $430 \mathrm{~s}$. The accompanying sensitivity was $82 \%(59 \%-100 \%)$ and the specificity was $89 \%$ (68\%-100\%). These results demonstrated that endotoxin administration prolongs $99 \mathrm{mTc}$-labeled mebrofenin excretion time.

\section{Liver ischemia-reperfusion injury prolongs hepatic excretion of $99 \mathrm{mTc}$-labeled mebrofenin}

Ischemic- and sham-operated livers were histologically analyzed according to the scoring system as described by Suzuki et al. [19]. Sinusoidal congestion was more pronounced in the liver IRI group as compared to the sham group at 2 hours $(\mathrm{p}=0.03)$ as well as at 24 hours after reperfusion $(\mathrm{p}=0.001)$ (Figure $3 \mathrm{~A}$ and $3 \mathrm{E}$ ). Cytoplasmic vacuolization of hepatocytes was present in mice that underwent liver IRI, but also in mice that underwent a sham operation at 2 and 24 hours after reperfusion (both time points $\mathrm{p}=0.2$ ) (Figure $3 \mathrm{~B}$ ). Vacuolization was present in all three zones of the liver, but was most pronounced in IRI as well as in sham mice in hepatocytes of the periportal zone, which is in line with the literature [20]. Necrosis was only found in mice that underwent liver IRI. The highest necrosis scores were found at 24 hours after reperfusion (Figure 3C). Also the combination of all histological features as the total damage score was significantly different between liver IRI and sham at 2 hours $(p=0.01)$ and 24 hours after reperfusion $(\mathrm{p}<0.001)$ (Figure 3D). Liver injury was also assessed by increased serum ALT and AST levels which peaked at 4 hours post-reperfusion (Figure $3 \mathrm{~F}$ ).
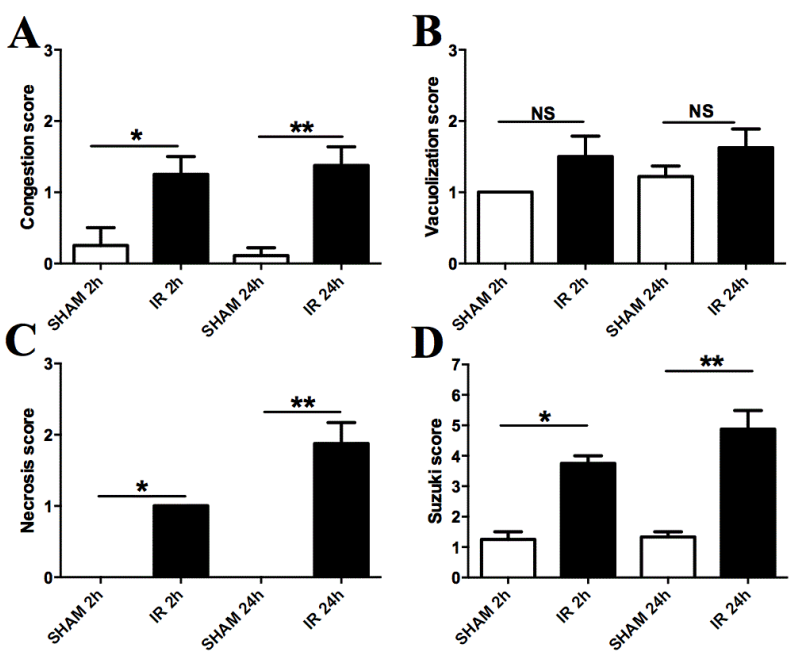

Figure 3: Liver pathology induced by ischemia/reperfusion. Scoring of sinusoidal congestion (A), hepatocyte vacuolization (B) and necrosis (C) in liver ischemia-reperfusion injury and sham operated mice 2 hours and 24 hours after reperfusion. (D) Suzuki score as composite parameter of sinusoidal congestion, hepatocyte vacuolization and necrosis in liver IRI- and sham-operated mice [19]. Each experimental group contains 6 mice. 
Citation: Sherer F, Van Simaeys G, Kers J, Yuan Q, Doumont G, et al. (2015) Dynamic Molecular Imaging for Hepatic Function Assessment in Mice: Evaluation in Endotoxin-Induced and Warm Ischemia-Reperfusion Models of Acute Liver Failure. J Liver 4: 170. doi: $10.4172 / 2167-0889.1000170$

Page 5 of 7

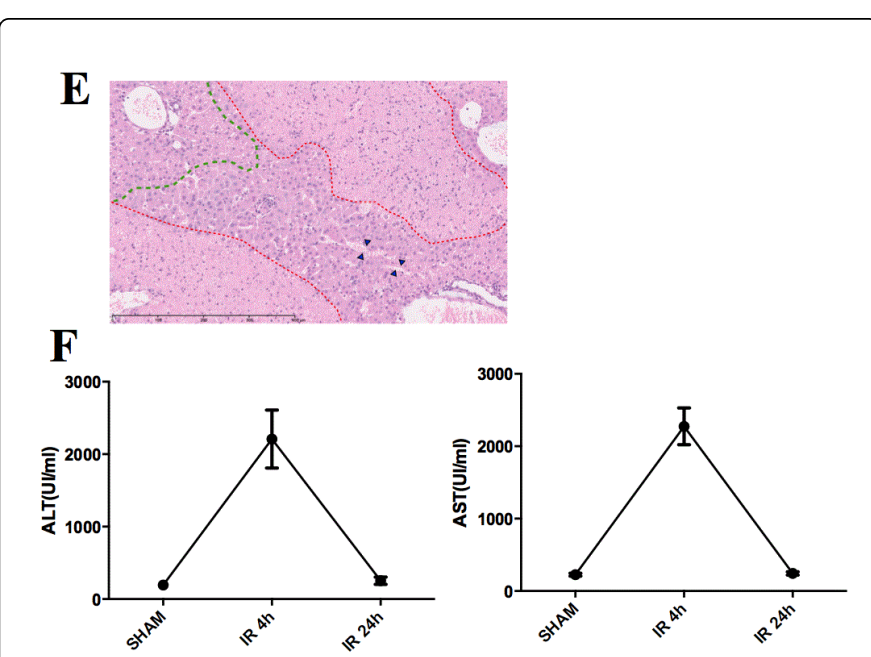

Figure 3: (E) Representative liver section stained with hematoxylin/ eosin showing venous congestion (blue arrowheads), vacuolization (green dotted area) and necrosis (red dotted areas) in an IR treated mouse at 24 hours. (F) Serum ALT and AST levels from C57BL/6 mice, 4 hours and 24 hours post reperfusion $(\mathrm{n}=8)$ and in SHAM vehicle controls $(n=6)$. Results are mean values \pm SEM. $\left({ }^{\star} \mathrm{p}<0.05\right.$, ${ }^{* *}$ $\mathrm{p}<0.01)$.

Imaging data showed that both characteristic excretion times (T0.5Exc and T0.2Exc) were significantly increased in $\mathrm{I} / \mathrm{R}$ mice at $2 \mathrm{H}$ after reperfusion compared with the sham animals (Figure $4 \mathrm{~A}$ and $\mathrm{B}$ ). Three animals never reached $50 \%$ of the maximum liver count value at the end of the scan in the IR2h group and one animal in the IR24h group. The T0.5Exc were $313 \mathrm{~s}$ (248-2296 s) [median (95\% CI)], $225 \mathrm{~s}$ (197-259 s) and $219 \mathrm{~s}(204-253 \mathrm{~s})$ in IR2h, sham and control animals, respectively ( $\mathrm{p}=0.01)$. The T0.2Exc were $738 \mathrm{~s}(577-3760 \mathrm{~s}), 619 \mathrm{~s}$ (464-671 s) and $538 \mathrm{~s}(471-586 \mathrm{~s})$ in IR2h, sham and control animals, respectively ( $\mathrm{p}=0.03$ ) (Figure $4 \mathrm{~A}$ and $\mathrm{B}$ ).

Individual excretion times are shown in Table 1. The CV for the T0.5Exc of liver IRI and sham subjected mice was $19 \%$ and $12 \%$ respectively while the $\mathrm{CV}$ for the T0.2Exc of liver IRI and sham subjected mice was $18 \%$ and $8 \%$ respectively, indicating that the T0.2Exc variable were slightly more reproducible in the liver IRI model. The ROC curve performed on T0.5Exc shows an area under de curve (AUC) of 0.92 (SE: 0.09, 95\% CI: 0.74-1.00), which proves to be statistically greater than 0.5 (1-tailed $\mathrm{p}<0.001)$ in IR2h versus sham animals (Figure 4C). The cut-off value for T0.5Exc was $260 \mathrm{~s}$. The accompanying sensitivity was $83 \%$ (95\% CI: $54 \%-100 \%)$ and the specificity was $100 \%$ (95\% CI: $100 \%-100 \%)$. A similar analysis performed on T0.2Exc gave an AUC of 0.75 (SE: 0.15, 95\% CI: 0.46-1.00), which proved to be statistically greater than 0.5 (1-tailed $\mathrm{p}=0.04)$. The cut-off value for T0.2Exc was $653 \mathrm{~s}$. The sensitivity was $67 \%(29 \%-100 \%)$ and the specificity was $83 \%(54 \%-100 \%)$ (Figure $4 \mathrm{D})$. At $24 \mathrm{~h}$ after reperfusion, the T0.5Exc were $206 \mathrm{~s}$ (154-732 s) [median (95\% CI)] and $185 \mathrm{~s}(148-217 \mathrm{~s})$ in IR24h and sham animals, respectively. The T0.2Exc were $448 \mathrm{~s}$ (350-1646 s) and $405 \mathrm{~s}$ (314-462 s) in IR24h and sham animals, respectively. At this time point, no difference in excretion rates reached statistical significance $(p=0.2)$
(Figure $4 \mathrm{E}$ and $4 \mathrm{~F}$ ). Since no statistically significant difference in excretion times between the IR24h group and sham-subjected animals was observed, ROC analyses was not performed. These results demonstrated that warm liver ischemia-reperfusion prolongs $99 \mathrm{mTc}$ labeled mebrofenin excretion time.
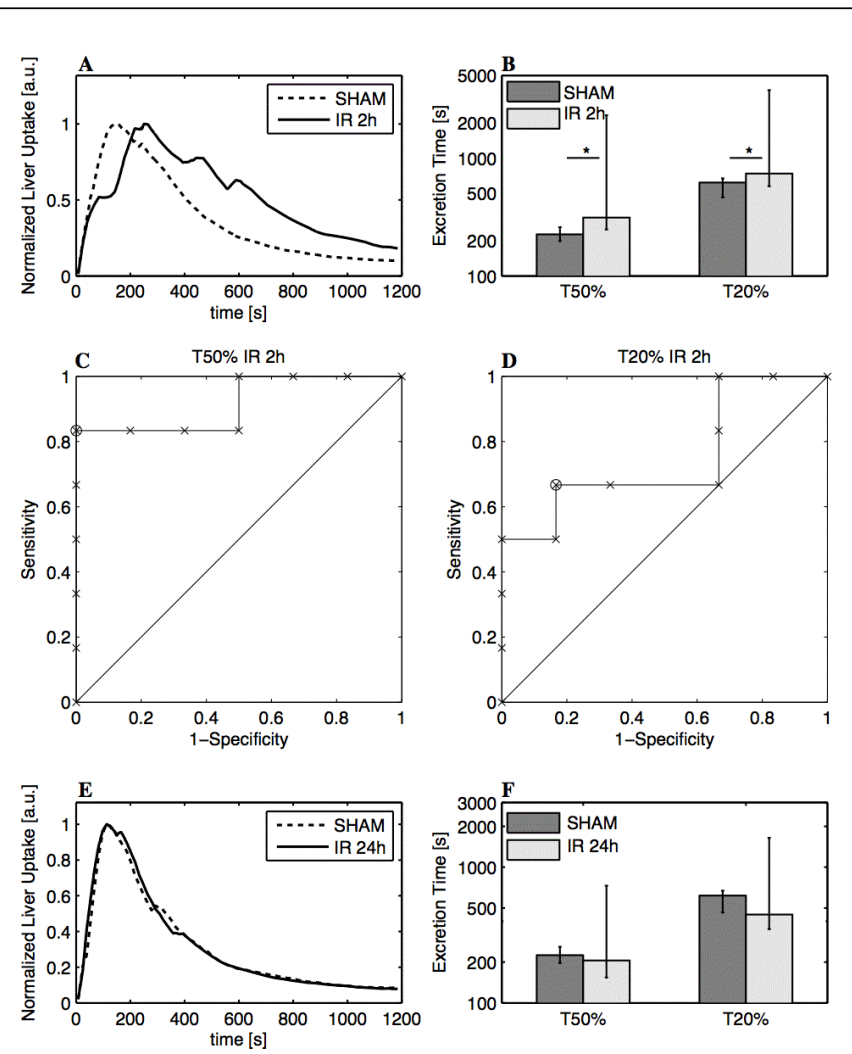

Figure 4: Liver excretion of $99 \mathrm{mTc}$-labeled mebrofenin after ischemia/reperfusion. (A) Excretion curves from IR mice (solid line, $n=6$ ) and SHAM operated mice (dashed line, $n=6$ ) obtained by taking the median value at each time point from the dynamic planar acquisitions, 2 hours post treatment, the arbitrary units were normalized (to 1 ) relative to the corresponding peak value in each group. (B) T0.5Exc and T0.2Exc values from IR treated mice $(n=6)$ and SHAM treated mice $(\mathrm{n}=6)$ at 2 hours. Results are median values $\pm 95 \%$ CI $\left(^{*} \mathrm{p}<0.05\right)$ (C) ROC curve for T0.5Exc for IR treated vs. SHAM treated mice. (D) ROC curve for T0.2Exc for IR treated vs. SHAM treated mice.T0.2Exc is the best to discriminate between IR treated mice and SHAM treated mice, 2 hours post treatment. (E) Excretion curves from IR mice (solid line, $n=6$ ) and SHAM operated mice (dashed line, $\mathrm{n}=4$ ) obtained by taking the median value at each time point from the dynamic planar acquisitions, 24 hours post treatment, the arbitrary units were normalized (to 1) relative to the corresponding peak value in each group. (F) T0.5Exc and T0.2Exc values from IR treated mice $(n=6)$ and SHAM treated mice $(n=4)$ at 24 hours. Results are median values $\pm 95 \% \mathrm{CI}$. 
Citation: Sherer F, Van Simaeys G, Kers J, Yuan Q, Doumont G, et al. (2015) Dynamic Molecular Imaging for Hepatic Function Assessment in Mice: Evaluation in Endotoxin-Induced and Warm Ischemia-Reperfusion Models of Acute Liver Failure. J Liver 4: 170. doi: $10.4172 / 2167-0889.1000170$

Page 6 of 7

\begin{tabular}{|c|c|c|c|c|c|c|c|c|c|c|c|c|c|c|}
\hline & \multicolumn{7}{|c|}{ T 0.5 EXC } & \multicolumn{7}{|c|}{ T 0.2 EXC } \\
\hline & \multicolumn{7}{|c|}{ Treatment } & \multicolumn{7}{|c|}{ Treatment } \\
\hline Mice & CTRL & $\begin{array}{l}\text { SHAM- } \\
\text { LPS }\end{array}$ & LPS & SHAM-2h & 1R-2h & $\begin{array}{l}\text { SHAM- } \\
24 \mathrm{~h}\end{array}$ & IR-24h & CTRL & $\begin{array}{l}\text { SHAM- } \\
\text { LPS }\end{array}$ & LPS & $\begin{array}{l}\text { SHAM- } \\
2 \mathrm{~h}\end{array}$ & IR- $2 h$ & $\begin{array}{l}\text { SHAM- } \\
24 \mathrm{~h}\end{array}$ & IR-24h \\
\hline 1 & 218.5 & 217 & 142 & 193 & 274.5 & 217 & 263 & 503 & 462 & 397 & 619 & 589 & 462 & 891 \\
\hline 2 & 219 & 198 & 156 & 200.5 & 2961 & 198 & 227 & 498 & 430 & 399 & 431 & 404 & 430 & 482 \\
\hline 3 & 205 & 266 & 171 & 259.5 & 301 & 148 & 1200 & 581 & 426 & 534 & 689 & 669 & 314 & 2400 \\
\hline 4 & 243 & 127 & 209 & 214 & 2296 & 172 & 184 & 573 & 311 & 477 & 496 & 3477 & 379 & 413 \\
\hline 5 & 204 & 131 & 234 & 235.5 & 324 & & 158 & 471 & 354 & 504 & 653 & 807 & & 373 \\
\hline 6 & 252.5 & 191 & 221 & 258 & 219.5 & & 150 & 586 & 401 & 440 & 619 & 567 & & 327 \\
\hline 7 & 162 & 131 & 224 & & & & & 426 & 301 & 577 & & & & \\
\hline 8 & & 180 & 1144 & & & & & & 417 & 1798 & & & & \\
\hline 9 & & 134 & 460 & & & & & & 344 & 1188 & & & & \\
\hline 10 & & & 271 & & & & & & & 768 & & & & \\
\hline 11 & & & 498 & & & & & & & 1345 & & & & \\
\hline Median & 219 & 180 & 224 & 225 & 312.5 & 185 & 205.5 & 538 & 401 & 534 & 618.5 & 738 & 404.5 & 448 \\
\hline $\begin{array}{l}\mathrm{Cl} \quad 95 \% \\
\text { Lower }\end{array}$ & 204 & 131 & 171 & 197 & 247.5 & 148 & 154 & 471 & 331.6 & 440 & 463.5 & 577 & 314 & 350 \\
\hline $\begin{array}{l}\mathrm{Cl} \quad 95 \% \\
\text { Upper }\end{array}$ & 253 & 207.5 & 460 & 259 & 2296 & 217 & 731.5 & 586 & 428 & 1188 & 671 & 3760 & 462 & 1646 \\
\hline
\end{tabular}

Table 1: Mebrofenin excretion times in the various groups

\section{Discussion}

This study showed the potential of $99 \mathrm{mTc}$-labeled mebrofenin scintigraphy for the evaluation of hepato-biliary function in murine models of liver dysfunction. During liver IRI, in particular in the liver transplantation context, the early immune phenomena that are activated within the injured tissue have a profound impact on the future of the damaged liver. Despite the introduction of new and effective immunosuppressive drugs, acute allograft rejection remains a major risk factor for long-term graft survival. The early biomarkers of liver injury that we have in hand at the present time provide indirect information. They assess, on the whole-organ scale, physiological activities (bilirubin excretion reflected by blood concentration) or signs of cellular stress or death (blood level of liver enzymes). Histological analysis of invasive core needle liver biopsies is the "gold standard" procedure for rejection diagnosis at the early posttransplantation phases. Liver biopsies provide precise but extremely local pathological information. This raises some doubt about the status of the whole organ since the insult may vary regionally within the organ. Liver biopsy is also an invasive procedure that bears the risk of rare but severe complications such as sepsis and hemorrhagic shock. The development and validation of non-invasive techniques, such as molecular in vivo imaging, would enable more accurate follow-up of the allograft considered in toto, and potentially avoid biopsies in many selected cases.
Non-invasive in vivo real-time monitoring of organ dysfunction has been proposed for hepatic IRI model assessment. Optical probes to monitor liver caspase-3 activity (i.e. apoptotic damage) [21] or realtime changes in intrahepatic NO concentration - a correlate of liver damage - using selective NO sensor have recently been proposed as alternatives to histological evaluation or hepatic enzyme measurement in IRI [22]. However, these methods are not exactly reflecting organ function failure, they provide very local information, and their translation into clinical practice might be difficult. We have developed a new dynamic imaging protocol of hepatic excretion with 99mTclabeled mebrofenin to assess organ dysfunction in murine models of experimental endotoxin-induced (septic) shock on one hand and liver ischemia-reperfusion injury - reflecting the transplantation surgery situation - on the other hand. The excretion rates of $99 \mathrm{mTc}$-labeled mebrofenin from the liver were significantly decreased in LPS-injected animals and in animals subjected to liver IRI.

In the clinics, $99 \mathrm{mTc}$-labeled mebrofenin has been mostly used so far for scintigraphic diagnosis of several biliary disorders such as cholecystitis, Oddi's sphincter dysfunctions and biliary leakage or fistula after hepato-biliary surgical procedures [10]. Hepatobiliary scintigraphy using iminodiacetic acid analogues has also been used in rat and dog models of acute and chronic liver disease [13,23]. Our study shows that $99 \mathrm{mTc}$-labeled mebrofenin may be exploited for quantitative analysis of liver dysfunction in mice. 
Citation: Sherer F, Van Simaeys G, Kers J, Yuan Q, Doumont G, et al. (2015) Dynamic Molecular Imaging for Hepatic Function Assessment in Mice: Evaluation in Endotoxin-Induced and Warm Ischemia-Reperfusion Models of Acute Liver Failure. J Liver 4: 170. doi: $10.4172 / 2167-0889.1000170$

Page 7 of 7

The decreased excretion rates of the $99 \mathrm{mTc}$-labeled mebrofenin from the liver in sick animals probably reflect damage not only to hepatocytes but also to endothelial cells [1,24]. Endothelial cell damage is indeed important to consider in this process because it has been shown to cause a reduction in hepatic perfusion slowing down tracer access to the hepatocytes [25]. The reduced excretion of the tracer in the bile canaliculi might therefore arise from combined hepatic hypoperfusion and reduced transport capacity of $99 \mathrm{mTc}$-labeled mebrofenin by damaged hepatocytes. Despite their localization in the space of Disse, damage of Kupffer cells and hepatic stellate cells might also be involved in the delayed recirculation and delivery of labeled mebrofenin to the hepatocytes. Indeed, these cell types are strongly affected in the LPS-induced liver inflammation model. Such a distinction might theoretically be accomplished by collecting data strictly related to regional liver perfusion. Liver perfusion imaging might involve various modalities (PET, SPECT, CT or MRI) that still need to be adapted to mice for this purpose.

Taken together, our results suggest that dynamic $99 \mathrm{mTc}$-labeled mebrofenin imaging is a potential method for assessing the severity of liver injury after ischemia-reperfusion and for the evaluation of interventions aiming at a reduction of this liver insult.

\section{Financial Support}

The Institute for Medical Immunology is sponsored by the government of the Walloon Region and GlaxoSmithKline Biologicals. The CMMI is supported by the European Regional Development Fund and Wallonia. This study was also supported by the Fonds National de la Recherche Scientifique (FNRS, Belgium) and an Interuniversity Attraction Pole of the Belgian Federal Science Policy. JK is financially supported by the European renal Association - European Renal and Transplant Association (ERA-EDTA) fellowship programme (grant number: ALTF 69-2010).

\section{References}

1. Teoh NC (2011) Hepatic ischemia reperfusion injury: Contemporary perspectives on pathogenic mechanisms and basis for hepatoprotectionthe good, bad and deadly. J Gastroenterol Hepatol 26 Suppl 1: 180-187.

2. Clavien PA, Petrowsky H, DeOliveira ML, Graf R (2007) Strategies for safer liver surgery and partial liver transplantation. N Engl J Med 356: 1545-1559.

3. Han C, Li G, Lim K, DeFrances MC, Gandhi CR, et al. (2008) Transgenic expression of cyclooxygenase- 2 in hepatocytes accelerates endotoxininduced acute liver failure. J Immunol 181: 8027-8035.

4. Jaeschke H, Woolbright BL (2012) Current strategies to minimize hepatic ischemia-reperfusion injury by targeting reactive oxygen species. Transplant Rev (Orlando) 26: 103-114.

5. Chen DL, Zhou D, Chu W, Herrbrich P, Engle JT, et al. (2012) Radiolabeled isatin binding to caspase-3 activation induced by anti-Fas antibody. Nucl Med Biol 39: 137-144.

6. de Graaf W, Häusler S, Heger M, van Ginhoven TM, van Cappellen G, et al. (2011) Transporters involved in the hepatic uptake of (99m)Tcmebrofenin and indocyanine green. J Hepatol 54: 738-745.

7. Gambhir SS, Hawkins RA, Huang SC, Hall TR, Busuttil RW, et al. (1989) Tracer kinetic modeling approaches for the quantification of hepatic function with technetium-99m DISIDA and scintigraphy. J Nucl Med 30: 1507-1518.
8. Ghibellini G, Leslie EM, Pollack GM, Brouwer KL (2008) Use of tc-99m mebrofenin as a clinical probe to assess altered hepatobiliary transport: integration of in vitro, pharmacokinetic modeling, and simulation studies. Pharm Res 25: 1851-1860.

9. Krishnamurthy S, Krishnamurthy GT (1989) Technetium-99miminodiacetic acid organic anions: review of biokinetics and clinical application in hepatology. Hepatology 9: 139-153.

10. Tulchinsky M (2010) The SNM practice guideline on hepatobiliary scintigraphy. J Nucl Med 51: 1825.

11. Veteläinen RL, Bennink RJ, de Bruin K, van Vliet A, van Gulik TM (2006) Hepatobiliary function assessed by $99 \mathrm{mTc}-$ mebrofenin cholescintigraphy in the evaluation of severity of steatosis in a rat model. Eur J Nucl Med Mol Imaging 33: 1107-1114.

12. Nunn AD, Loberg MD, Conley RA (1983) A structure-distributionrelationship approach leading to the development of Tc-99m mebrofenin: an improved cholescintigraphic agent. J Nucl Med 24: 423-430.

13. Daniel GB, DeNovo RC, Schultze AE, Schmidt D, Smith GT (1998) Hepatic extraction efficiency of technetium-99m-mebrofenin in the dog with toxic-induced acute liver disease. J Nucl Med 39: 1286-1292.

14. Yüksel M, Hatipoglu A, Temiz E, Salihoglu YS, Hüseyinova G, et al. (2000) The role of hepatobiliary scintigraphy in the evaluation of the protective effects of dimethylsulphoxide in ischaemic/reperfusion injury of liver. Nucl Med Commun 21: 775-780.

15. [No authors listed] (2007) Making the most of mouse models. Nat Immunol 8: 657 .

16. Neyt S, Huisman MT, Vanhove C, De Man H, Vliegen M, et al. (2013) In vivo visualization and quantification of (Disturbed) Oatp-mediated hepatic uptake and Mrp2-mediated biliary excretion of $99 \mathrm{mTc}$ mebrofenin in mice. J Nucl Med 54: 624-630.

17. West MA, Keller GA, Hyland BJ, Cerra FB, Simmons RL (1985) Hepatocyte function in sepsis: Kupffer cells mediate a biphasic protein synthesis response in hepatocytes after exposure to endotoxin or killed Escherichia coli. Surgery 98: 388-395.

18. Lentsch AB, Yoshidome H, Cheadle WG, Miller FN, Edwards MJ (1998) Chemokine involvement in hepatic ischemia/reperfusion injury in mice: roles for macrophage inflammatory protein-2 and KC. Hepatology 27: 1172-1177.

19. Suzuki S, Toledo-Pereyra LH, Rodriguez FJ, Cejalvo D (1993) Neutrophil infiltration as an important factor in liver ischemia and reperfusion injury. Modulating effects of FK506 and cyclosporine. Transplantation 55: $1265-1272$

20. Taniai H, Hines IN, Bharwani S, Maloney RE, Nimura Y, et al. (2004) Susceptibility of murine periportal hepatocytes to hypoxiareoxygenation: role for NO and Kupffer cell-derived oxidants. Hepatology 39: 1544-1552.

21. Ozaki M, Haga S, Ozawa T (2012) In vivo monitoring of liver damage using caspase-3 probe. Theranostics 2: 207-214.

22. Nakagawa A, Yokoyama Y, Suzuki H, Shoji K, Watanabe Y, et al. (2012) Real-time monitoring of liver damage during experimental ischaemiareperfusion using a nitric oxide sensor. Br J Surg 99: 1120-1128.

23. Malhi H, Bhargava KK, Afriyie MO, Volenberg I, Schilsky ML, et al. (2002) $99 \mathrm{mTc}$-mebrofenin scintigraphy for evaluating liver disease in a rat model of Wilson's disease. J Nucl Med 43: 246-252.

24. Guiral JS, Hinson JA, Farhood A, Jaeschke H (2004) NADPH oxidasederived oxidant stress is critical for neutrophil cytotoxicity during endotoxemia. Am J Physiol Gastrointest Liver Physiol 287: G243-252.

25. Vollmar B, Menger MD (2009) The hepatic microcirculation: mechanistic contributions and therapeutic targets in liver injury and repair. Physiol Rev 89: 1269-1339. 